\title{
A Non-CT Based Total Knee Arthroplasty System Featuring Complete Soft-Tissue Balancing
}

\author{
Manuela Kunz ${ }^{1}$, Matthias Strauss ${ }^{2}$, Frank Langlotz ${ }^{1}$, Georg Deuretzbacher ${ }^{2}$, \\ Wolfgang Rüther ${ }^{2}$, and Lutz-Peter Nolte ${ }^{1}$ \\ 1 M.E. Müller Institute for Biomechanics, University of Bern, Switzerland \\ \{Manuela.Kunz, Frank.Langlotz, Lutz.Nolte\}@memot.unibe.ch, \\ http://cranium. unibe.ch \\ 2 Department of Orthopaedics, University Hospital Hamburg-Eppendorf, Germany \\ \{strauss, deuretzbacher\}@uke.uni-hamburg.de
}

\begin{abstract}
The postoperative result of a total knee arthroplasty strongly depends on surgical performance. Since the classical instrumentation does not allow the surgeon to find the optimal positions of components in any situation and to perform an adequate ligament balancing, we developed a non-CT based computer-assisted Total Knee Arthroplasty system. The system contains four main parts: the anatomy registration, the soft-tissue balancing navigation, the planning, and the resections navigation part. The registration of the patient anatomy allows the alignment of the prosthesis component according to the mechanical axis. Beside this, the implementation of soft tissue balancing and intraoperative planning is required for a good postoperative kinematics result. We collected early experiences in various in-vivo and in-vitro tests, where the average postoperative Varus/Valgus angle was 1.8 degree.
\end{abstract}

\section{Introduction}

The aim of each total knee arthroplasty is to return the patient to a more active and pain-free lifestyle. One major mechanism for such a successful longtime result is the alignment of the prosthesis components according to the mechanical limb axes (1], 5]), which requires a precise intra-operative registration of these axes. Both, malalignment and non-obtaining of an ideal knee kinematics are reasons for a failure of total knee arthroplasty $([1$, 5, 7]). The essential requirements for a good postoperative kinematics situation are an adequate ligament balancing and a stable and non-restricted range of motion (ROM), which results from balanced gap distances in flexion and extension. Additionally to the tibio-femoral stability, stability between femur and patellar is required for a successful kinematics result. The latter may be achieved by restoring the natural joint line. Of course the outcome of the total knee arthroplasty depends on the postoperative care and the patient compliance but the basis is the intra-operative activity of the surgeon. We developed a computer assisted total knee arthroplasty system to help the surgeon archieving greater accuracy during the intraoperative procedure. 


\section{Methods}

An optical tracking device is placed in the operating theater to track relative motions between femur, tibia, and instruments. For this purpose dynamic reference bases are attached to each of these objects.

The first step during the intraoperative usage of the system is the registration of the patient's anatomy using the technique of "surgeon defined anatomy", as it was introduced in the area of computer assisted total knee arthroplasty by Leitner et.al in 1997 (4, [5], 7]). Additionally ligament behavior is taken into account. This allows to navigate soft-tissue balancing and to include an intra-operative planning step, which contains both, a manual and an automatic planning option. Finally the planned resection planes are executed by means of adapted cutting jigs.

\section{Registration of the Patient's Anatomy}

For successful navigation during total knee replacement, the axes of femur and tibia (defined by the centers of hip, femoral knee, tibia knee, and ankle) as well as certain geometric features of the knee have to be determined. For the hip center registration a pivoting method is used, similar to the one presented by Piccard et.al ([6]). However, we implemented an alternative to track possible pelvis motion by using a pointer placed onto the spina iliaca rather than an invasivly fixated reference base. During the rotation of the femur the system records the orientation of the femur and the position of the pointer tip and calculates the pivoting center according to these values. The registrations of the knee centers and the ankle center are based on anatomical structures, which are intraoperativly digitized by the surgeon using specially designed instruments. On the femoral knee side the surgeon palpates the attachment areas of the collateral ligaments. The system determines the most prominent points within these areas and calculates the epicondylar femoral axis based on its finding. The center of this axis marks a reference point for the latero-medial location of the femoral knee center. Depending on the prosthesis design this center can be corrected anterior-posteriorly towards the center of the femoral shaft, which is calculated by palpated points on the ventral and dorsal cortexes. The tuberosity and eminentia are the reference landmarks taken for the tibial knee center. One of the measurements for the ankle center calculation is the transmalleolar axis. The surgeon defines it by digitalization of both malleoli. For the location of the ankle center on the transmalleolar axis the surgeon identifies an additional reference like the ligament tibiales anterior or then second toe.

Additionally digitized landmarks are used for the calculation of the anatomically best fitting component size, the defect-management, the restoring of the natural joint line, and the synchronization of the natural tibial rotation and the postoperative rotation of the tibial component. 


\section{Intraoperative Planning and Soft-Tissue Balancing}

The registration of the patient's anatomy allows alignment of the component according to the mechanical axes, but obtaining an ideal knee kinematics requires an intraoperative planning step. The major aims of this planning step are

1. Creation of a uniform extension gap

2. Creation of a uniform flexion gap

3. Balancing the distances of both gaps

To create a uniform extension gap the soft tissue behavior has to be taken into account by loading both collateral ligaments, which can be performed in two different ways, either by manually stressing the knee in varus/valgus directions or by using distractors that hold both collateral ligaments under the same tension. Based on this recorded ligament behavior the system displays the varus/valgus angle between the femoral and tibial mechanical axes. The surgeon can use this information to perform a soft tissue balancing. In case that it is not possible to completely align the extension gap by manipulating the soft tissues, the system offers to modify the varus or valgus angle of the planed femoral distal and/or tibial resection plane. This allows the creation of a stable knee in extension even in difficult preoperative knee situations.

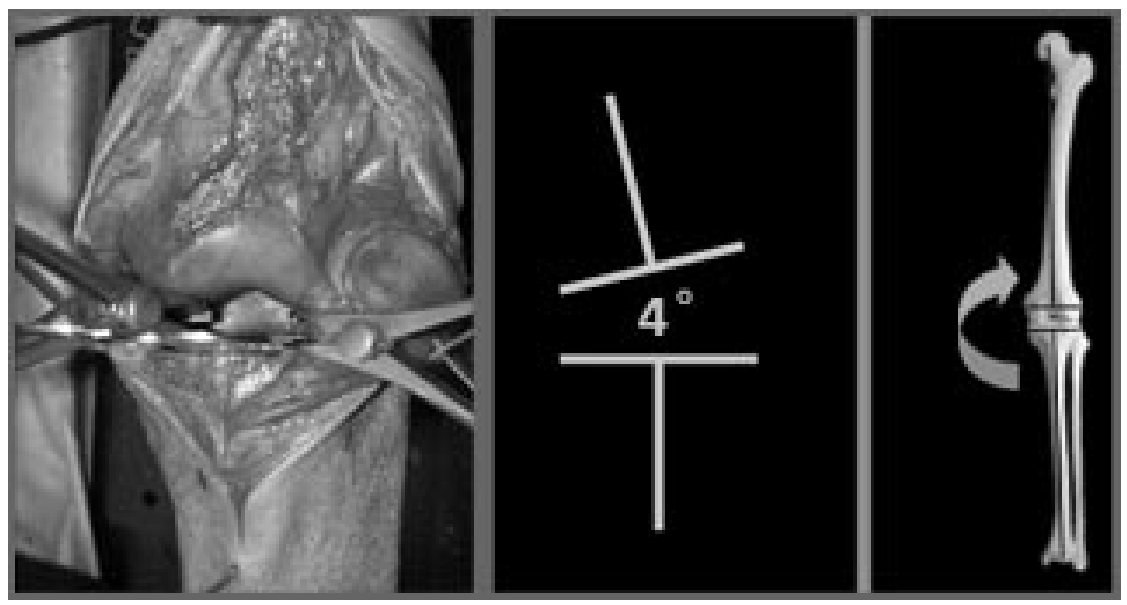

Fig. 1. Soft tissue balancing in extension. Left side: Use of distrators to bring collateral ligaments in tension. Right side: The system displays varus or valgus angle to help the surgeon performing soft tissue balancing.

For the following planning step the final ligament behavior in both extension and flexion will be taken into account by the same procedure, which was 
described before. The recorded information about the ligament behavior in flexion is used in the intraoperative planning step to define the outside rotation of the femoral component. The result of the patient-depend determination of the outside rotation is a postoperatively stable flexion gap. The planned situation now features uniform extension and flexion gaps. However, both gaps have to be balanced for a successful arthroplasty. For this purpose additional manual planning features are implemented. To facilitate the complex adjustment of the considerable number of parameters, we introduced an automatic planning step, which proposes an initial planning situation in the following manner: The flex-

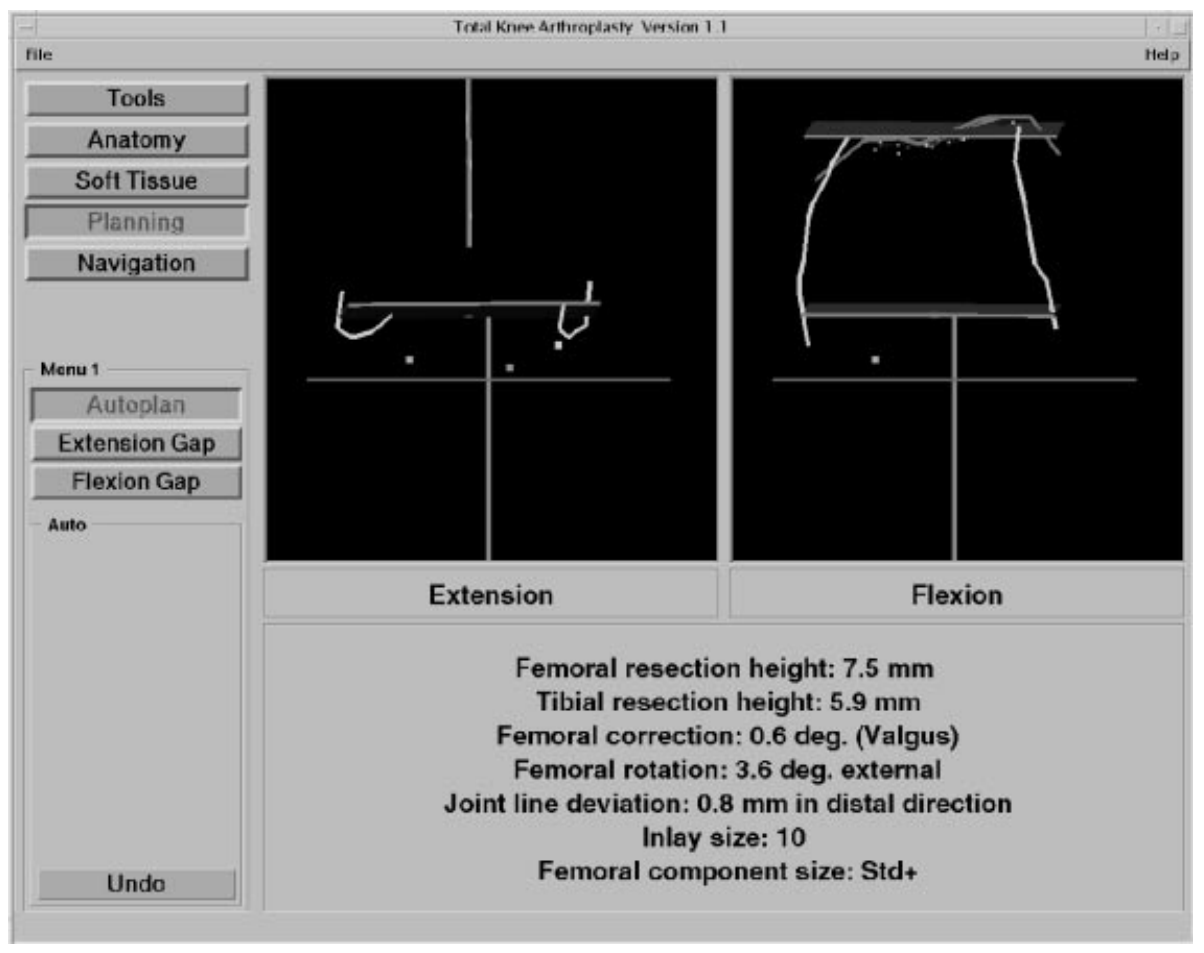

Fig. 2. Auto-Planner after calculation. The system suggests optimal positioning of the prosthesis components and simulates numerically and graphically the resulting postoperative situation in extension and flexion.

ion gap is defined based on the recorded ligament behavior, the planned femoral component size, and a predefined minimal tibial resection height. According to the recorded ligament situation in extension the transfer of this flexion gap into extension results the femoral distal resection height. Next the system gives information about the deviation of the planned joint line from the natural joint line and navigates the surgeon to up- or downsize the femoral component. As a con- 
sequence this modification of the flexion gap changes also the planned femoral resection height for a better restoring of the natural joint line. Figure 2 shows the in interface for such a autoplanning step.

The outcome of the automatic planning can be used by the surgeon as an initial situation for the manually planning. This allows both, a fast intraoperative planning without loosing of surgery time and the including of the surgeon's experience into the plan. After the surgeon has performed the planning step the system helps with a graphical interface to accurately perform the planned resection planes. For this purpose the conventional cutting jigs are equipped with LED's. The system presents the deviation between the target plane and the real resection plane in different views.

\section{Results}

We collected early experiences with the system in a number of in-vivo and invitro tests. Table 1 summarizes the results of a study, which was performed on three fresh frozen cadaver specimens. This kind of specimen-preperation also allowed us to test the soft-tissue balancing in a realistic situation. During this process we performed the qualitative evaluation with trial prostheses and measured also the postoperative instability in extension.

Table 1. First results of in-vitro tests

\begin{tabular}{|c|c|c|c|c|c|}
\hline \multirow{2}{*}{ Nr. } & \multicolumn{4}{|c|}{ Passive Extension } & \multirow{2}{*}{ Varus/Valgus Stress } \\
\cline { 2 - 5 } & Coronal & \multicolumn{2}{|c|}{ Sagittal } & \multirow{2}{*}{ Post. Instability } \\
\cline { 2 - 5 } & Pre & Post & Pre & Post & \\
\hline \hline 1 & $0^{\circ}$ & $-1.5^{\circ}$ & $0.1^{\circ}$ Varus & $0.5^{\circ}$ Valgus & $1.5^{\circ}$ Varus \\
\hline 2 & $-14.0^{\circ}$ & $-1.5^{\circ}$ & $1.5^{\circ}$ Varus & $1.1^{\circ}$ Valgus & $1.7^{\circ}$ Varus \\
\hline 3 & $-10.0^{\circ}$ & $+0.6^{\circ}$ & $8.3^{\circ}$ Varus & $0.5^{\circ}$ Valgus & $0.7^{\circ}$ Varus \\
\hline
\end{tabular}

Table 2 lists the postoperative data of 10 patients, who recieved a total knee arthroplasty with the help of our system. The values were measured directly after the surgery, whithout loading. For measuring Varus/Valgus angle in passive extension postoperative radiographs were used. The varus valgus stress was determined with help of the system.

The presented values are the result of a first clinical trial phase of the system. We are now going to evaluate the system by performing full clinical studies. 
Table 2. First results of in-vivo tests

\begin{tabular}{|c|c|c|}
\hline \multicolumn{2}{|c|}{ Passive Extension } & Varus/Valgus Stress \\
\hline Coronal & Sagittal & Post. Instability \\
\hline \hline $0.8^{\circ}-4.2^{\circ}\left(\right.$ av. $\left.1.9^{\circ}\right)$ & $0.2^{\circ}-3.9^{\circ}\left(\right.$ av. $\left.1.8^{\circ}\right)$ & Av. $7.2^{\circ}$ \\
\hline
\end{tabular}

\section{Conclusions}

The presented system is based on the registration of the mechanical limb axes, which does not require a CT scan, equivalent to the systems presented by Piccard et al., Krakow et al (3], [4, [5, 7]), and Ritschl et al. ([2]). In order to realize accurate results the calculation of the mechanical axes must not depend on anatomical landmarks, which are influenced by the pathological situation of the knee. In addition to respecting the bony anatomy we introduced the soft tissue consideration into the intraoperative planning step, which allows producing a postoperative result, that is optimized regarding anatomy and kinematics. For this purpose also the anterior and posterior femoral resection planes are navigated by the system.

\section{References}

1. Andriacchi, T.P.: Dynamics of Knee Malalignment. Orthopaedic Clinics of North America 25(3) (1994) 395-403

2. Ritschl, P., Fuiko, R., Broers, H., Wurzinger, A., Berner, W.: Computerassisted navigation and robot-cutting system for total knee replacement. In Proceedings of the 1st Annual Meeting of the International Society for Computer Assisted Orthopaedics Surgery, Davos (2001) 94

3. Krackow, K., Bayers-Thering, M., Phillips, M., Mihalko, W.: A new technique for determining proper mechanical axis alignment during total knee arthroplasty: progress toward computer-assisted TKA. Orthopedics 22(7) (1999) 698-702

4. Leitner, F., Picard, F., Minfelde, R., Schulz, H.-J., Cinquin, P., Saragaglia, D.: Computer-Assited Knee Surgical Total Replacement. In CVDMed-MRCAS '97. J. Troccaz, E. Grimson, and R. Moesger, editors. Springer Verlag, Berlin Heidelberg New York (1997) 629-637

5. Miehlke, R.K., Clemens, U., Kershally, S.: Computer Integrated Instrumentation in Knee Arthroplasty - A comparative study of conventinal and computerized Technique. In CAOS USA 2000 - Conference Proceeding, June 15-17, 2000, Pittsburgh, Pennsylvania, USA. A.M. DiGioia and L.P. Nolte, editors. Centers for Medical Robotics and Computer Assisted Surgery, Western Pennsylvania Institute for Computer Assisted Surgery, Pittsburgh (2000) 93-96

6. Moreland, J.R.: Mechanisms of Failure in Total Knee Arthroplasty. Clinical Orthopaedics and Related Research 226 (1987) 49-63

7. Picard, F., Leitner, F., Saragaglia, D., Raoult, O., Chaussard, C., Montbarbon, E.: Intraoperative Navigation for TKA: Location of a rotational center of the knee 
and hip. In CAOS USA 2000 - Conference Proceeding, June 15-17, 2000, Pittsburgh, Pennsylvania, USA. A.M. DiGioia and L.P. Nolte, editors. Centers for Medical Robotics and Computer Assisted Surgery, Western Pennsylvania Institute for Computer Assisted Surgery, Pittsburgh (2000) 91-92.

8. Sathasivam, S. Walker, P.S.: The conflicting requirements of laxity and conformity in total knee replacement. Journal of Biomechanics 32 (1999) 239-247 